INPLASY

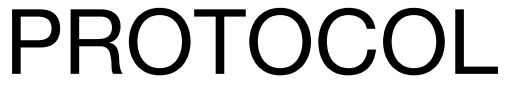

To cite: Cai et al. Effects of different dosages of SodiumGlucose Transporter 2 Inhibitors on lipid levels in patients with type 2 diabetes mellitus: a protocol for systematic review and metaanalysis. Inplasy protocol 202040201. doi:

10.37766/inplasy2020.4.0201

Received: 28 April 2020

Published: 28 April 2020

Corresponding author: TingYu Cai

tingyucai2018@126.com

Author Affiliation:

Hospital of Chengdu

University of Traditional

Chinese Medicine

Support: None.

Review Stage at time of this submission: The review has not yet started.

Conflicts of interest: None.

\section{Effects of different dosages of Sodium-Glucose Transporter 2 Inhibitors on lipid levels in patients with type 2 diabetes mellitus: a protocol for systematic review and meta-analysis}

Cai, T1 Zhang, L2; Yang, T33 Gao, Y4.

Review question / Objective: The effect of SGLT2 inhibitors on lipid levels was inconsistent in different studies. Although statistical analyses by relevant meta-analyses and systematic reviews have shown that SGLT2 inhibitors are associated with an augment in HDL and LDL cholesterol, the latest findings are not incorporated and evidence of a dose-related association is insufficient. The purpose of this systematic review and meta-analysis is to evaluate the association and degree of association between different doses of SGLT2 inhibitors and changes in blood lipid levels in patients with type 2 diabetes, in order to provide a reliable basis for clinical medication.

Condition being studied: SGLT-2 inhibitors, which are widely applied in the management of type 2 diabetes, have shown favourable effects on glycemic control. Up to now, there have been many studies on SGLT2 inhibitors, and there have also been relevant reports on its effect on blood lipid level of diabetic patients. However, the results of each study were not completely consistent, and there was no specific evaluation of the association.

INPLASY registration number: This protocol was registered with the International Platform of Registered Systematic Review and Meta-Analysis Protocols (INPLASY) on 28 April 2020 and was last updated on 28 April 2020 (registration number INPLASY202040201).

\section{INTRODUCTION}

Review question / Objective: The effect of SGLT2 inhibitors on lipid levels was inconsistent in different studies. Although statistical analyses by relevant metaanalyses and systematic reviews have shown that SGLT2 inhibitors are associated with an augment in HDL and LDL cholesterol, the latest findings are not incorporated and evidence of a doserelated association is insufficient. The purpose of this systematic review and meta-analysis is to evaluate the association and degree of association between different doses of SGLT2 inhibitors and changes in blood lipid levels in patients 
with type 2 diabetes, in order to provide a reliable basis for clinical medication.

Condition being studied: SGLT-2 inhibitors, which are widely applied in the management of type 2 diabetes, have shown favourable effects on glycemic control. Up to now, there have been many studies on SGLT2 inhibitors, and there have also been relevant reports on its effect on blood lipid level of diabetic patients. However, the results of each study were not completely consistent, and there was no specific evaluation of the association.

\section{METHODS}

Participant or population: Participants diagnosed with type 2 diabetes aged $\geq 18$ years will be included in the study, irrespective of race and sex, severity of illness and other factors.

Intervention: Trails whose therapeutic agents are SGLT2 inhibitors (Canagliflozin, Dapagliflozin, Empagliflozin, Ertugliflozin, Ipragliflozin, Luseogliflozin, Tofogliflozin) and a minimum study duration of 12 weeks are eligible.

Comparator: Controls contain placebo and other hypoglycemic drugs, such as metformin, sulfonylureas (SUs), ningestedglinide, thiazolidinediones (TZDs), a-glucosidase inhibitors (AGI), dipeptidyl peptidase-IV (DPP-IV) inhibitors, glucagonlike peptide 1 (GLP-1) receptor agonist and insulin.

Study designs to be included: All clinical randomized controlled trials on humans of SGLT2 inhibitors treatment in type 2 diabetes mellitus will be collected.

Eligibility criteria: Participants age $\geq 18$ years with a diagnosis of type 2 diabetes. The minimal study duration is 12 weeks. The dosage of the SGLT2 inhibitors and Lipid levels(TC,TG,LDL-C,HDL-C, etc.) at baseline and after treatment were reported.

Information sources: Relevant studies will be identified by a literature search of PubMed, EMBASE, the Web of Science,
The Cochrane Library, China National Knowledge Infrastructure (CNKI), Chinese Biological Medical literature Database (CBM), Chinese VIP Information (VIP) and Wan Fang Database from database inception to March 31,2020 . The search is divided into three conceptual groups. One group includes the term used to describe a "sodium-glucose transporter 2 inhibitor," another encompasses the terminology with regard to "type 2 diabetes mellitus," and the third contains a "randomized controlled trial". We will retrieve Medical Subject Headings (MeSH) and equivalent control terms and keywords in all databases aforementioned.

Main outcome(s): Lipid changes from baseline in different SGLT2 inhibitors dosage groups and control groups will be tested. We will investigate whether different categories of SGLT2 inhibitors have different effects on lipid levels. The intensity of association between SGLT2 inhibitors dosage and lipid change.

Quality assessment / Risk of bias analysis: Each original study will be independently evaluated for risk of bias by two authors under the guidance of the Cochrane Handbook for Systematic Reviews of Interventions. The authors will assess seven domains associated with the risk of bias in each study, including random sequence generation, allocation concealment, blinding (blinding of participants and researchers, and blinding of outcome assessment), incomplete outcome data, selective reporting the outcomes and other bias. And then, the evaluation results will be classified as low risk, high risk and unclear risk. Unresolved discrepancies will be consulted with the third author.

Strategy of data synthesis: We will design a data extraction table to specify the data scope to be included. The data extraction table contains the following information in each study: basic information of literature (title, first author and contact information, publication year), study characteristics (study design, sample size, number in each group, randomization method, blinding), 
participants characteristics (race, age, sex, duration of disease, etc.), intervening characteristics (intervention measures, dose, frequency, duration of treatment), curative effects and outcomes data. The authors independently performed preextraction the files after prior training in relevant data extraction. On the basis of pre-extraction, the shortcomings of extraction table will be found and corrected. All included studies are going to be extracted according to the final data extraction table. If sufficient data information is not available in the literature, more detailed data will be obtained by contacting the corresponding author via email or telephone. The two authors resolve their differences through discussion. If no agreement can be reached, a final decision will be made in consultation with the third author.

Subgroup analysis: If heterogeneity is found to be substantial, we will conduct subgroup analysis to identify the source of inter-study heterogeneity. Referring to the new criteria for evaluating the credibility of subgroup analyses, 20 we hypothesized several subgroups based on possible influencing factors: race, age, sex, course of the disease, drug combination, etc.

Sensibility analysis: Sensitivity analysis will be performed to determine the robustness of the meta-analysis results. We will eliminate some of the low-level ambiguities and then reaggregate and analyze the data to compare the differences between the reworked results and the original results. In addition, it is possible to reanalyze the data using different statistical methods, such as using the random-effects model instead of the fixed-effects model.

\section{Country(ies) involved: China.}

Keywords: Sodium-Glucose Transporter 2 Inhibitors, type 2 diabetes mellitus, lipid, protocol, systematic review and metaanalysis.

Contributions of each author:

Author 1 - TingYu Cai - Author 1 drafted the manuscript.
Author 2 - Li Zhang - The author provided statistical expertise.

Author 3 - Ting Yang - The author contributed to the development of the selection criteria, and the risk of bias assessment strategy.

Author 4 - Yang Gao - The author read, provided feedback and approved the final manuscript. 